\title{
PDGF regulates chondrocyte proliferation through activation of the GIT1- and PLC $\gamma 1$-mediated ERK1/2 signaling pathway
}

\author{
JIN XIAO, XUQIONG CHEN, LIPENG XU, YING ZHANG, QINGSHUI YIN and FEI WANG \\ Department of Orthopedics, Liuhuaqiao Hospital, Guangzhou, Guangdong 510010, P.R. China
}

Received September 23, 2013; Accepted March 17, 2014

DOI: $10.3892 / \mathrm{mmr} .2014 .2506$

\begin{abstract}
Studies investigating the effects of cytokines on chondrocytes have significant application potential, since the culture of cartilage cells in vitro is a vital step for cartilage tissue engineering. Platelet-derived growth factor (PDGF), one of the growth factors occurring at the early stage of the healing process of damaged tissue, is critical in bone healing. The present study investigated the effects of the activation of PDGF on cell proliferation, apoptosis and the underlying mechanisms of chondrocytes in vitro. The results indicated that the stimulation of PDGF led to overexpression of the G-protein-coupled receptor kinase interacting protein-1 (GIT1) and promotion of the phosphorylation of phospholipase $\mathrm{C} \gamma 1$ (PLC $\gamma 1)$. Furthermore, PDGF induced chondrocyte proliferation and inhibited apoptosis via activation of the extracellular signal-regulated kinase (ERK) 1/2 pathway. Following knocking down GIT1 expression by small interfering RNA, phosphorylation of PLC $\gamma 1$ and activation of the ERK1/2 pathway was no longer promoted by PDGF. In addition, the effects of PDGF on proliferation and apoptosis were suppressed. The expression levels of GIT1 were not affected; however, the phosphorylation of ERK1/2 was suppressed through inhibition of the phosphorylation of PLC $\gamma 1$ by U73122. The results demonstrated that GIT1 is upstream of PLC $\gamma 1$. Although the ability of PDGF to induce cell proliferation was inhibited by the inhibition of the ERK1/2 pathway by PD98059, apoptosis was not suppressed. In conclusion, the present study demonstrated that PDGF was able to activate the GIT1-PLC $\gamma 1$-mediated ERK1/2 pathway to control chondrocyte proliferation.
\end{abstract}

Correspondence to: Dr Ying Zhang, Department of Orthopedics, Liuhuaqiao Hospital, 111 Liuhua Avenue, Guangzhou, Guangdong 510010, P.R. China

E-mail: zhangying_doc@aliyuu.com

Key words: G-protein-coupled receptor kinase interacting protein-1, chondrocyte, phospholipase $\mathrm{C} \gamma 1$, platelet-derived growth factor, extracellular signal-regulated kinase $1 / 2$, proliferation

\section{Introduction}

The biological repair of articular cartilage defects is a focus of the study of orthopedics and tissue engineering is generally considered to be the most promising and effective approach $(1,2)$. The isolation and cell culture of articular chondrocytes in vitro aims to obtain a sufficient amount of the desired seed cells for cartilage tissue engineering (2). Furthermore, the application of cytokines aims to accelerate cell proliferation and maintain the normal function of articular cartilage cells (2). Therefore, investigation concerning the mechanisms of action of cytokines in cultured chondrocytes in vitro has significant application potential.

Platelet-derived growth factor (PDGF), a $30 \mathrm{kDa}$ dimer of $\mathrm{A}$ and $\mathrm{B}$ polypeptide chains linked by disulphide bonds, is a multiple mitogen for the regulation of the replication of osteoblasts and degradation of bone collagens $(3,4)$. A study by Brandl et al (2) demonstrated that there was a markedly higher proliferation rate of chondrocytes cultured with PDGF-BB and transforming growth factor (TGF)- $\beta 1$ than that of the control cells. Schmidt et al (4) revealed that PDGF was able to promote chondrocyte proliferation and proteoglycan synthesis. It has also been reported that PDGF is able to stimulate cultured chondrocytes and repair cartilage tissue proliferation $(5,6)$. PDGF was demonstrated to be the autocrine cytokine of human osteoblasts (3). Therefore, the present study focused on its effects on chondrocyte formation.

To date, few studies have investigated the specific mechanisms underlying the action of PDGF in cartilage cells. Rui et al (7) revealed that the overexpression of G-protein-coupled receptor kinase interacting protein-1 (GIT1) induced by PDGF was able to activate the extracellular signal-regulated kinase (ERK) $1 / 2$ pathway to promote osteoblast migration and bone healing. GIT1, a multidomain scaffold protein, is involved in the internalization and membrane transportation of the $G$ protein-coupled receptor (8). Pang et al (8) demonstrated that the interaction between mitogen-activated protein kinase (MAPK)-1 and GIT1 was able to activate ERK1/2. Additionally, the translocation of ERK1/2 and activation in focal adhesions are responsible for cell spreading and migration with the binding of GIT1 $(7,8)$. Certain studies have demonstrated that GIT1 is expressed in osteoblasts and osteoclasts, implying that GIT1 may be involved in bone metabolism $(7,9,10)$. 
Additionally, even though there were no significant effects on the differentiation and function of mouse osteoblasts in the absence of GIT1, the GIT1 Y321F mutant was able to inhibit PDGF-induced osteoblast movement and migration, according to the Boyden chamber assay $(7,10)$. The studies suggested that the phosphorylation of tyrosine 321 of GIT1 is closely associated with the combination of focal adhesion kinase and the migration of osteoblasts induced by PDGF $(7,10)$. The formation of pseudopodia bodies in osteoclasts without GIT1 resulted in abnormal bone resorption and destruction capabilities and affected the regulation of the osteoclast cytoskeleton receptor activator of nuclear factor $-\kappa \mathrm{B}$.

In addition, GIT1, apart from interacting with ERK-1 and activating ERK1/2, is also essential for the phosphorylation of phospholipase C $\gamma 1$ (PLC $\gamma 1)(8,11)$. Furthermore, Ren et al (11) revealed that cyclic mechanical stress may promote PLC $\gamma 1$ phosphorylation and activate the ERK1/2 signaling pathway for rat chondrocyte proliferation and extracellular matrix synthesis. PLC $\gamma 1$, one of the serine threonine kinases of PLC, is important in the regulation of various cellular responses generated by stimuli in the signal transduction pathway (12). Husain et al (13) demonstrated that the proliferative activity of endothelial cells during overexpression of vascular endothelial growth factor receptor-2 depended on the activation of PLC $\gamma 1$. Hunter et al (14) demonstrated that PDGF activated the PLC $\gamma 1$-mediated ERK1/2 signaling pathway and ultimately regulated the transcription of vascular smooth muscle cells (14). Thus, in the present study, it was hypothesized that PDGF is able to activate the ERK1/2 pathway for the regulation of chondrocyte proliferation and apoptosis through the promotion of GIT1 expression and PLC $\gamma 1$ phosphorylation. As a result, therefore, the aim of the present study was to verify the above hypothesis at the cellular level, based on the isolation and cell culture of chondrocytes.

\section{Materials and methods}

Materials. Sprague-Dawley neonatal rats were purchased from the Institute of Laboratory Animal Sciences, Chinese Academy of Medical Sciences and Peking Union Medical College (Beijing, China). The present study was approved by the ethics committee of Liuhuaqiao Hospital (Guangzhou, China). Dulbecco's modified Eagle's medium (DMEM) was obtained from Invitrogen Life Technologies (Carlsbad, CA, USA). The MEK1/2 inhibitor PD98059 was purchased from Cell Signaling Technology (Beverly, MA, USA) and the PLC $\gamma 1$ inhibitor U73122 was obtained from Santa Cruz Biotechnology, Inc. (Santa Cruz, CA, USA). The whole antibodies used in the present study were purchased from Cell Signaling Technology and thiazolyl blue tetrazolium bromide and propidium iodide (PI) were purchased from Sigma-Aldrich (St. Louis, MO, USA).

Cell culture. One-week-old neonatal Sprague-Dawley rats were dissected and their limb joints were extracted under sterile conditions. The fibrous tissue of the articular cartilage surface was stripped carefully and the cartilage was cut from the transparent central portion into pieces of $\sim 1 \mathrm{~mm}^{3}$ in size. The tissue pieces were incubated with trypsin $(0.25 \%)$ at $37^{\circ} \mathrm{C}$ for $30 \mathrm{~min}$. The supernatant was discarded following centrifugation at $10,000 \mathrm{x}$ g for $5 \mathrm{~min}$. Centrifugation using a 200 mesh filter and at $10,000 \mathrm{x}$ g for $5 \mathrm{~min}$ was performed to obtain the pellet. Following that, $0.2 \%$ collagenase II was added for digestion and incubated at $37^{\circ} \mathrm{C}$ for $4 \mathrm{~h}$. Finally, the cells were maintained in DMEM containing FBS $(10 \%)$, penicillin $(100 \mathrm{U} / \mathrm{ml})$ and streptomycin $(50 \mathrm{U} / \mathrm{ml})$ at $37^{\circ} \mathrm{C}$ in a humidified incubator with $5 \% \mathrm{CO}_{2}$, respectively. The morphology of the cells purified by repeated isolation and culture was observed under an inverted phase contrast microscope. Once the cells had grown completely in the dish, $0.25 \%$ trypsin was used for digestion and passage. Then, $10^{5}$ cultured cartilage cells were collected and seeded in a six-well plate. The cells were rinsed with phosphate-buffered saline (PBS) when they were adherent. Following fixing with $4 \%$ paraformaldehyde at $4^{\circ} \mathrm{C}$ for $30 \mathrm{~min}$, the conventional avidin-biotin complex method for type II collagen staining was performed and the stained cells were observed under an Olympus IX-70 microscope (Olympus, Tokyo, Japan).

Transfection of small interfering (si) RNA. The cells were seeded at a density of $1 \times 10^{5}$ cells $/ \mathrm{ml}$ into a six-well plate and incubated for $24 \mathrm{~h}$. siRNA transfection was performed according to the manufacturer's instructions of Lipofectamine 2000 (Invitrogen Life Technologies), when the cell confluence reached $\sim 70 \%$. The siRNA sequences used were: GIT1 forward, 5'-AAGCTGCCAAGAAGAAGCTAC-3' and reverse, 5'-AATTCTCCGACACGTGTCACT-3'. GIT1 siRNA was prepared and transfected at $100 \mathrm{nM}$ for $24 \mathrm{~h}$ as previously described (4,5). The MEK1/2 inhibitor PD98059 and PLC $\gamma 1$ inhibitor U73122 were dissolved in dimethyl sulfoxide (DMSO; Sigma-Aldrich). After the cells were pretreated with PD98059 (30 nM), U73122 $(1 \mu \mathrm{M})$ or the same volume of $0.1 \%$ DMSO for $1 \mathrm{~h}, 10 \mathrm{ng} / \mathrm{ml}$ of PDGF (Sigma-Aldrich) was added to stimulate for $24 \mathrm{~h}$. Following $72 \mathrm{~h}$, transfected cells were analyzed by quantitative polymerase chain reaction (qPCR) and western blot analysis.

Western blot analysis. The lysis buffer was used for the extraction of total cellular protein from the cells. Following adding lysis buffer, the mixture was agitated at $4{ }^{\circ} \mathrm{C}$ for $20 \mathrm{~min}$. The protein in the cell lysates was collected from the supernatant following centrifugation at $10,000 \mathrm{x}$ g at $4^{\circ} \mathrm{C}$ for $10 \mathrm{~min}$. The protein concentrations in the cell lysates were determined by the Bradford protein assay. SDS-PAGE was conducted in $8 \%$ glycine gels (Bio-Rad, Hercules, CA, USA) loading equal amounts of proteins per lane. Following electrophoresis, the nitrocellulose membrane was used for transformation to separate proteins. Then, the nitrocellulose membrane was inhibited with 5\% non-fat milk in Tween 20 with Tris-buffered saline (TBST) buffer for $1 \mathrm{~h}$. Following that, the membranes were incubated with primary antibodies at a dilution of $1: 1,000$ overnight at $4^{\circ} \mathrm{C}$ and then anti-rabbit immunoglobulin $\mathrm{G}$ monoclonal antibody conjugated with horseradish peroxidase (Cell Signaling Technology) at a dilution of 1:7,000-8,000 was incubated for $1 \mathrm{~h}$ at $37^{\circ} \mathrm{C}$. TBST was used for washing the membranes every $10 \mathrm{~min}$, for a total of $30 \mathrm{~min}$. The protein bands were detected using enhanced chemoluminescence. The light-emitting films were scanned by a GelBlot-Pro 1.01 gel imaging system (UVP, Inc., Upland, 
CA, USA) for western blotting and gray values of the bands were measured by Gel-Pro Analyzer software 6.3 (Media Cybernetics, Rockville, MD, USA).

Bromodeoxyuridine (BrdU) cell proliferation assay. To examine the roles of PDGF, PLC $\gamma 1$, GIT1 and the ERK1/2 pathway on cell proliferation, the effects of different inhibitors on cell proliferation were examined by a BrdU assay. Briefly, the cells were seeded into a six-well plate and incubated for $24 \mathrm{~h}$, and then siRNA transfection was performed using Lipofectamine 2000. Following pretreatment of the cells with $50 \mu \mathrm{M}$ NSC23766, $30 \mathrm{nM}$ PD98059 or the same volume of $0.1 \%$ DMSO for $1 \mathrm{~h}, 10 \mathrm{ng} / \mathrm{ml}$ PDGF was then added to stimulate the cells for $24 \mathrm{~h}$. A BrdU colorimetric immunoassay kit (Cell Proliferation ELISA; Roche Diagnostics, Mannheim, Germany) was used for the quantification of cell proliferation according to the manufacturer's instructions. The cell proliferation was expressed as the mean percentage of the control values (set at $100 \%$ ).

Flow cytometric analysis. The cells were trypsinized and washed with PBS. Following that, the cells were fixed with $75 \%$ ethanol overnight at $-20^{\circ} \mathrm{C}$. The next day, PBS was used to wash the fixed cells. Following that, propidium iodide (PI) working solution $(1.21 \mathrm{mg} / \mathrm{ml}$ Tris; $700 \mathrm{U} / \mathrm{ml}$ RNase; $50.1 \mu \mathrm{g} / \mathrm{ml} \mathrm{PI}$; pH 8.0) was stained with the fixed cells for $4 \mathrm{~h}$ in the dark. An Epics XL-MCL flow cytometer (Beckman Coulter, Miami, FL, USA) was used to analyze the stained cells. The cell cycle distribution was analyzed using MultiCycle software 5.0 (Phoenix Flow Systems, San Diego, CA, USA). The proportion of cells in G0/G1, S and G2/M phases are presented as DNA histograms. The apoptotic cells with hypodiploid DNA contents were measured by quantifying the sub-G1 peak in the cell cycle pattern. For each experiment, 10,000 events per sample were recorded.

Terminal deoxynucleotidyl transferase dUTP nick end labeling (TUNEL)-4',6-diamidino-2-phenylindole (DAPI) co-staining assay. Apoptotic cells were assessed by a TUNEL-DAPI co-staining assay using an In Situ Cell Death Detection kit (Roche Diagnostics) according to the manufacturer's instructions. Briefly, cultured cells were washed with PBS and then fixed with $4 \%$ formaldehyde at $4^{\circ} \mathrm{C}$ for $25 \mathrm{~min}$. The cells were then incubated with $0.2 \%$ Triton X-100 for $5 \mathrm{~min}$. Following incubation with $100 \mu \mathrm{l}$ of equilibration buffer at room temperature for $10 \mathrm{~min}$, the cells were mixed with $50 \mu \mathrm{l}$ of TUNEL reaction mixture containing nucleotide mixture and terminal deoxynucleotidyl transferase for $60 \mathrm{~min}$ at $37^{\circ} \mathrm{C}$. The cells were washed with $2 \mathrm{X}$ saline sodium citrate for $15 \mathrm{~min}$. The cells were then incubated with $0.3 \% \mathrm{H}_{2} \mathrm{O}_{2}$ for $10 \mathrm{~min}$ and with streptavidin working solution for $30 \mathrm{~min}$ at room temperature, respectively. Then, $0.5 \mu \mathrm{g} / \mathrm{ml}$ of DAPI was added and cells were incubated in a humidified chamber in the dark for $5 \mathrm{~min}$ at room temperature. Finally, they were examined and images were captured under a fluorescence microscope (Nikon Eclipse 80i; Nikon, Tokyo, Japan).

Statistical analysis. The experiments were performed at least in triplicate and the results are expressed as the mean \pm standard deviation. SPSS statistical package (SPSS 13.0 for

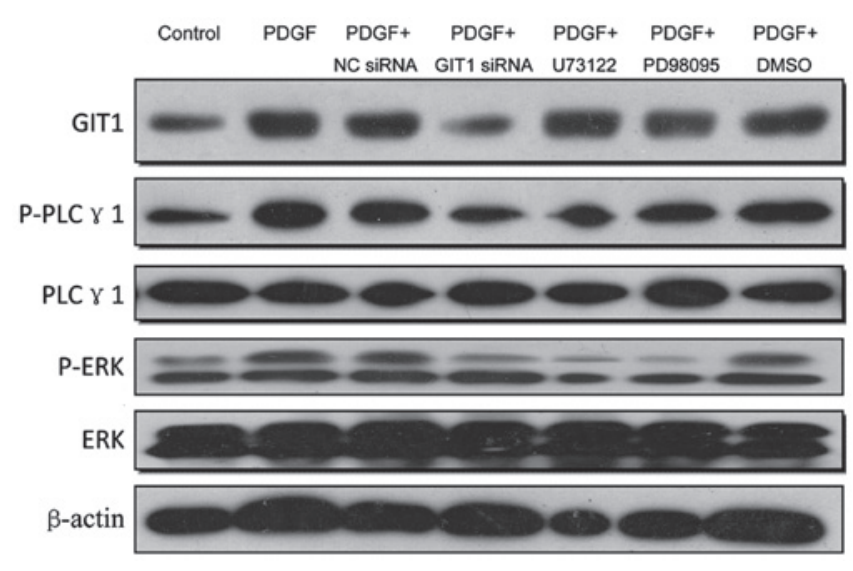

Figure 1. PDGF increases the expression of GIT1 to promote the phosphorylation of PLC $\gamma 1$ and ERK. Following pretreatment of the cells with siRNA transfection, $50 \mu \mathrm{M}$ NSC23766, $30 \mathrm{nM}$ PD98059 or the same volume of DMSO for $1 \mathrm{~h}, 10 \mathrm{ng} / \mathrm{ml}$ PDGF was then added to stimulate for $24 \mathrm{~h}$. PDGF, platelet-derived growth factor; GIT1, G-protein-coupled receptor kinase interacting protein-1; P-PLC $\gamma 1$, phosphorylated phospholipase $C \gamma 1$; ERK, extracellular signal-regulated kinase; DMSO, dimethyl sulfoxide; siRNA, small interfering RNA.

Windows; SPSS, Inc., Chicago, IL, USA) was used for statistical analysis. The difference between two groups was analyzed by a two-tailed Student's t-test and that between three or more groups was analyzed by one-way analysis of variance multiple comparisons. ${ }^{*} \mathrm{P}<0.05$ or ${ }^{* *} \mathrm{P}<0.01$ was considered to indicate a statistically significant difference.

\section{Results}

PDGF increases the expression of GIT1 to promote the phosphorylation of PLC $\gamma 1$ and ERK. In the present study, the effects of PDGF, GIT1, PLC $\gamma 1$, ERK1/2 and their crosstalk in chondrocytes were investigated by siRNA silencing techniques and western blot analysis. The cells were seeded and cultured in a six-well plate for $24 \mathrm{~h}$, followed by siRNA transfection of GIT1 siRNA using Lipofectamine 2000. Following pretreatments of the cells with siRNA transfection or NSC23766,PD98059 and DMSO for $1 \mathrm{~h}, 10 \mathrm{ng} / \mathrm{ml}$ of PDGF was then added to stimulate the cells for $24 \mathrm{~h}$. The expression levels of associated proteins were examined by western blot analysis. As shown in Fig. 1, the results demonstrated that PDGF was able to increase the expression of GIT1 by 2.8-fold, thereby promoting the phosphorylation of PLC $\gamma 1$ and activation of the ERK1/2 pathway by 3.0 and 4.0 -fold, respectively. In the group in which GIT1 was depleted by siRNA, even though PDGF was added to the chondrocytes for stimulation, the expression levels of GIT1 decreased to $60 \%$ compared with those in the control. In addition, the PLC $\gamma 1$ inhibitor U73122 or the ERK1/2 pathway inhibitor PD98059 did not affect GIT1 expression significantly, suggesting that GIT1 was upstream of PLC $\gamma 1$. Furthermore, PDGF stimulation was able to induce the overexpression of PLC $\gamma 1$ and the phosphorylation of ERK1/2. However, PDGF did not markedly increase the expression of PLC $\gamma 1$ and the phosphorylation of ERK1/2 when GIT1 expression was inhibited. The results demonstrated that PDGF was not able to stimulate the upregulation of ERK1/2 due to the 
suppression of PLC $\gamma 1$. Therefore, PDGF first upregulated the expression of GIT1, then activated PLC $\gamma$ and eventually activated the ERK1/2 pathway.

Roles of PLC $\gamma 1$, GIT1 and ERK1/2 in PDGF-activated cell proliferation. To further examine the biological functional roles of PLC $\gamma 1$, GIT1 and ERK1/2 in chondrocytes, the effects of inhibition of PDGF-activated cell proliferation by RNA interference or small molecule inhibitors were investigated. Briefly, the cells were seeded into a six-well plate and incubated for $24 \mathrm{~h}$, and then GIT1 siRNA transfection was performed using Lipofectamine 2000. Following pretreatment of the cells with siRNA transfection, $50 \mu \mathrm{M}$ NSC23766, $30 \mathrm{nM}$ PD98059 or the same volume of $0.1 \%$ DMSO for $1 \mathrm{~h}, 10 \mathrm{ng} / \mathrm{ml}$ PDGF was then added to stimulate the cells for $24 \mathrm{~h}$, and then the cell proliferation was examined by a BrdU assay. As shown in Fig. 2, PDGF was able to promote the cell proliferation of chondrocytes through the GIT1- and PLC $\gamma$-mediated ERK1/2 pathway and GIT1 may be essential in the activation of PLC $\gamma$ and ERK1/2. The investigation of the effects of the ERK1/2 inhibitor PD98059 and PLC $\gamma$ inhibitor U73122 on chondrocyte proliferation verified that chondrocyte proliferation was significantly decreased by inhibition of either PLC $\gamma$ or ERK1/2. Based on these results, it is possible that there are certain other pathways involved in the induction of chondrocyte proliferation by PDGF. At present, there are no studies comparing the effects of inhibiting the phosphorylation of PLC $\gamma$ and the inhibition of the expression of ERK1/2. Chondrocyte proliferation was increased by PDGF when PLC $\gamma$ phosphorylation or ERK1/2 expression were inhibited, indicating that additional proteins may be involved in PDGF-induced growth stimulation.

PDGF affects the cell cycle of chondrocytes. The effects of GIT1 knockdown on cell cycle distribution were analyzed by flow cytometry. Cell cycle analysis by quantitation of DNA content was one of the earliest applications of flow cytometry. The DNA of mammalian cells is able to be stained by a variety of DNA binding dyes, including PI. The premise with these dyes is that they are stoichiometric i.e. they bind in proportion to the amount of DNA present in the cell. In this way cells that are in the S phase have more DNA than cells in G1 and, therefore, they take up proportionally more dye and fluoresce more brightly until they have doubled their DNA content. Cells in G2 phase incorporate approximately twice as much stain as cells in G1 phase. As shown in Fig. 3, following depletion of GIT1, the number of cells in G0/G1 phase increased significantly from 32.4 (control) to $52.1 \%$, with a slight decrease of cells in $\mathrm{S}$ phase. This inhibition of cell cycle progression explains the observed decreases in cell proliferation.

Effect of PLC $\gamma 1$, GIT1 and ERK1/2 on apoptosis. Apoptosis is the process of programmed cell death that occurs in multicellular organisms. It has been verified that apoptosis in chondrocytes is important for bone remodeling (15). The TUNEL-DAPI assay is a common method for detecting DNA fragmentation that results from apoptotic signaling cascades. The assay relies on the presence of nicks in the DNA which are able to be identified by the TUNEL assay, which was used to elucidate the role of GIT1 in chondrocyte apoptosis in the

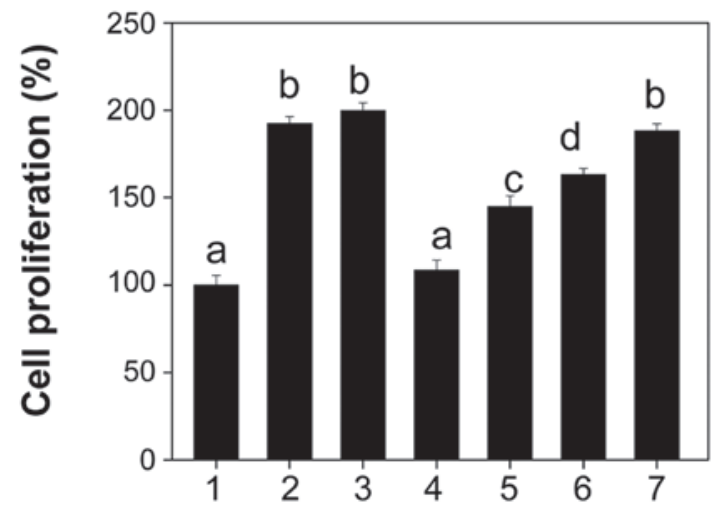

Figure 2. Roles of PLC $\gamma 1$, GIT1 and ERK1/2 in PDGF-activated cell proliferation. Following pretreatment of the cells with siRNA transfection, $50 \mu \mathrm{M}$ NSC23766, $30 \mathrm{nM}$ PD98059 or the same volume of $0.1 \%$ DMSO for $1 \mathrm{~h}, 10 \mathrm{ng} / \mathrm{ml}$ PDGF was then added to stimulate for $24 \mathrm{~h}$. The cell proliferation was examined by BrdU assay. 1, Control; 2, PDGF; 3, PDGF + NC siRNA; 4, PDGF + GIT1 siRNA; 5, PDGF + U73122; 6, PDGF + PD98095; 7, PDGF + DMSO. PDGF, platelet-derived growth factor; GIT1, G-protein-coupled receptor kinase interacting protein-1; PLC $\gamma 1$, phospholipase $\mathrm{C} \gamma 1$; ERK, extracellular signal-regulated kinase; DMSO, dimethyl sulfoxide; siRNA, small interfering RNA; NC, negative control; BrdU, bromodeoxyuridine. a, b, c and d indicate a statistically significant difference between each other $(\mathrm{P}<0.01)$.

present study. The results (Fig. 4) demonstrated that apoptosis, as evidenced by DNA fragmentation, was observed in chondrocytes. Notably, the addition of PDGF was able to effectively inhibit the occurrence of apoptotic cell death. Furthermore, depletion of GIT1 and PLC $\gamma 1$ effectively reversed the protective effects of PDGF. However, the inhibition of ERK1/2 demonstrated no significant effect on apoptosis. Taken together, these results suggested that GIT1 and PLC $\gamma 1$ are necessary for the survival of chondrocytes induced by PDGF, while the ERK1/2 pathway was not important in the anti-apoptotic effect of PDGF on chondrocytes.

\section{Discussion}

PDGF, one of the growth factors involved in the early stage of healing of damaged tissue, is an essential growth factor for the healing process $(16,17)$. PDGF is able to stimulate cartilage DNA and protein synthesis (18). PDGF is also known to increase the IGF1 production of mesenchymal cells and was able to stimulate chondrocyte proteoglycan synthesis by IGF1 (19). At present, there have been few studies investigating the complete mechanisms of PDGF in cartilage cells.

According to the studies of Rui et al (7) and Ren et al (11), the present study hypothesized that PDGF is able to activate the ERK1/2 pathway for the regulation of chondrocyte proliferation and apoptosis through the promotion of GIT1 expression and PLC $\gamma 1$ phosphorylation. In the present study, PDGF was used to stimulate the cultured chondrocytes in vitro to investigate its effects. The results indicated that GIT1 was overexpressed and PLC $\gamma 1$ and ERK1/2 were induced to undergo phosphorylation. It was revealed that the induction of PLC $\gamma 1$ and ERK1/2 phosphorylation was not significant when PDGF was knocked down by siRNA. In other words, GIT1 was involved in the ERK1/2 pathway activated by PDGF and was able to activate PLC $\gamma 1$ and ERK1/2 phosphorylation. 


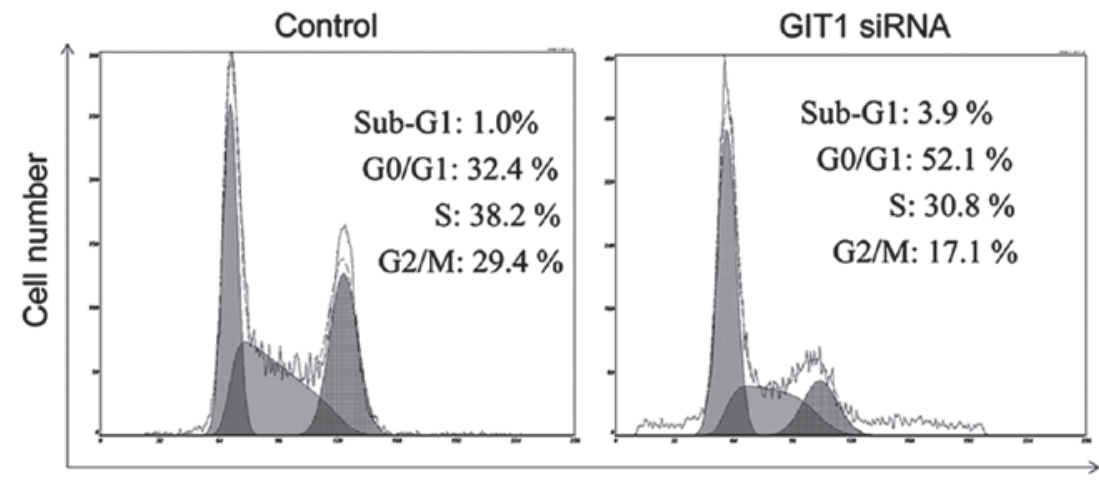

DNA content

Figure 3. Effects of GIT1 deletion on cell cycle distribution. Following transfection of the cells with siRNA and incubation for another $24 \mathrm{~h}$, cell cycle distribution was analyzed by flow cytometry. GIT1, G-protein-coupled receptor kinase interacting protein-1; siRNA, small interfering RNA.

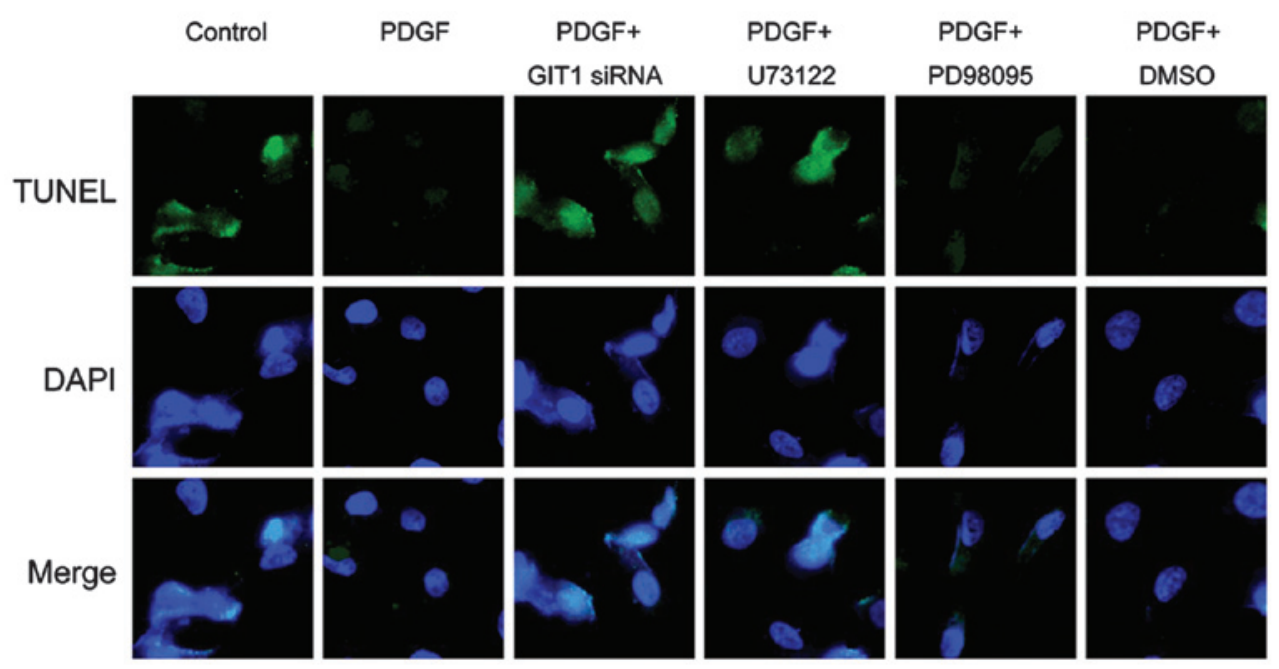

Figure 4. Effects of GIT1, PLC $\gamma 1$ and ERK1/2 on the anti-apoptotic activity of PDGF in chondrocytes. Following pretreatment of the cells with siRNA transfection and various specific inhibitors, $10 \mathrm{ng} / \mathrm{ml}$ PDGF was then added to stimulate for $24 \mathrm{~h}$. Apoptosis was determined by a TUNEL-DAPI co-staining assay. PDGF, platelet-derived growth factor; GIT1, G-protein-coupled receptor kinase interacting protein-1; PLC $\gamma 1$, phospholipase C $\gamma 1$; ERK, extracellular signal-regulated kinase; TUNEL, terminal deoxynucleotidyl transferase dUTP nick end labeling; DAPI, 4'6-diamidino-2-phenylindole; DMSO, dimethyl sulfoxide; siRNA, small interfering RNA.

Following the application of the PLC $\gamma 1$ inhibitor U73122 and the ERK1/2 inhibitor PD98059, the expression of GIT1 did not alter significantly, suggesting that GIT1 is upstream of PLC $\gamma 1$. In addition, activation of the ERK1/2 signaling pathway induced by PDGF was not significant when PLC $\gamma 1$ was inhibited by U73122. Thus, activation of the ERK1/2 signaling pathway by PDGF proceeds via upregulated expression of PDGF, activation of GIT1 followed by activation of PLC $\gamma 1$, and eventually the activation of the ERK1/2 pathway. This is consistent with the hypothesis of the present study.

Based on the results on the cell proliferation of chondrocytes, PDGF stimulated the proliferation of chondrocytes, which was markedly attenuated following the downregulation of GIT1. The ability of PDGF to promote proliferation was also inhibited when PLC $\gamma 1$ phosphorylation and the ERK1/2 pathway were blocked. However, the inhibitory effect on cell proliferation by inhibition of the ERK1/2 pathway was lower than the effect of inhibition of GIT1 and PLC $\gamma 1$. These results revealed that GIT1 and PLC $\gamma 1$ may promote chondrocyte proliferation through other pathways. The study by Ren et al (11) verified that GIT1, as a scaffold protein, is closely associated with PLC $\gamma 1$ and the ERK1/2 pathway. Furthermore, GIT1 was able to control the regulation of chondrocyte proliferation, migration and apoptosis through the PLC $\gamma 1$ and ERK1/2 pathway (20). Based on studies on osteoblasts, PDGF is vital in osteoblast proliferation and differentiation and is able to promote osteoblast DNA synthesis at an early stage, leading to the transition of osteoblasts from stationary G0/G1 into the replicative S phase. It also enhances the migration of monocytes and fibroblasts, so that local fibroblast proliferation and differentiation can be induced to promote bone formation. Yang et al (7) demonstrated that the number of osteoblasts in G0/G1 phase was reduced, while that in the $\mathrm{S}$ phase was increased in human fetal skull cells in vitro. In addition, PDGF-AA, PDGF-BB and TGF were able to enhance the expression of PDGF-A mRNA (7). This demonstrated that PDGF-AA is able to accelerate the cell cycle and induce the division of quiescent cells to enhance cell replication, thereby promoting bone formation. Based on the results of a space study by Kumei et al (20), PDGF- $\beta$, epidermal growth factor and its receptor binding are able to be adjusted by the microgravity in 
space caused by decreased osteoblast function, and are able to activate tyrosine kinases to bind with the adapter protein Shc Grb2. The induction of Ras/MAPK is able to stimulate signaling through different chemical and physical reactions to the nucleus, leading to the induction of c-fos and c-jun gene expression (20).

The present study demonstrated that PDGF stimulation reduced chondrocyte apoptosis. However, downregulation of GIT1 expression and inhibition of the phosphorylation of PLC $\gamma 1$ is able to inhibit the effects of PDGF. By contrast, inhibition of the ERK1/2 pathway did not significantly affect chondrocyte apoptosis. Previous studies have identified GIT1 as a regulator of mitochondrial biogenesis and function, which is required for postnatal cardiac maturation (21). The present study also demonstrated that GIT1 is involved in the proliferation of chondrocytes, which is an important process for fracture healing. Furthermore, a study by Zhang et al (22) indicated that GIT1 inhibited apoptosis via the modulation of the inositol triphosphate receptor-mediated $\mathrm{Ca}^{2+}$ signal (22). Therefore, the suppression of chondrocyte apoptosis by PDGF was mediated by the promotion of the expression of GIT1 and the phosphorylation of PLC $\gamma 1$; however, it did not proceed via the ERK1/2 pathway.

In conclusion, PDGF was able to promote chondrocyte proliferation and inhibit apoptosis through the promotion of the expression of GIT1 and the phosphorylation of PLC $\gamma 1$. The stimulation of proliferation by PDGF was achieved through the ERK1/2 pathway; however, the results indicated that other pathways were also involved. By contrast, the suppression of apoptosis by PDGF did not proceed via the ERK1/2 pathway.

\section{References}

1. Filardo G, Kon E, Di Martino A, Iacono F and Marcacci M Arthroscopic second-generation autologous chondrocyte implantation: a prospective 7-year follow-up study. Am J Sports Med 39: 2153-2160, 2011.

2. Brandl A, Angele P, Roll C, Prantl L, Kujat R and Kinner B: Influence of the growth factors PDGF-BB, TGF-betal and bFGF on the replicative aging of human articular chondrocytes during in vitro expansion. J Orthop Res 28: 354-360, 2010.

3. Yang D, Chen J, Jing Z and Jin D: Platelet-derived growth factor (PDGF)-AA: a self-imposed cytokine in the proliferation of human fetal osteoblasts. Cytokine 12: 1271-1274, 2000.

4. Schmidt MB, Chen EH and Lynch SE: A review of the effects of insulin-like growth factor and platelet derived growth factor on in vivo cartilage healing and repair. Osteoarthritis Cartilage 14: 403-412, 2006.

5. Barbero A, Palumberi V, Wagner B, Sader R, Grote MJ and Martin I: Experimental and mathematical study of the influence of growth factors on the growth kinetics of adult human articular chondrocytes. J Cell Physiol 204: 830-838, 2005.
6. Dorotka R, Windberger U, Macfelda K, Bindreiter U, Toma C and Nehrer S: Repair of articular cartilage defects treated by microfracture and a three-dimensional collagen matrix. Biomaterials 26: 3617-3629, 2005.

7. Rui Z, Li X, Fan J, et al: GIT1Y321 phosphorylation is required for ERK1/2- and PDGF-dependent VEGF secretion from osteoblasts to promote angiogenesis and bone healing. Int J Mol Med 30: 819-825, 2012.

8. Pang J, Xu X, Wang X, et al: G-protein-coupled receptor kinase interacting protein-1 mediates intima formation by regulating vascular smooth muscle proliferation, apoptosis, and migration. Arterioscler Thromb Vasc Biol 33: 999-1005, 2013.

9. Menon P, Yin G, Smolock EM, Zuscik MJ, Yan C and Berk BC: GPCR kinase 2 interacting protein 1 (GIT1) regulates osteoclast function and bone mass. J Cell Physiol 225: 777-785, 2010.

10. Ren Y, Yu L, Fan J, et al: Phosphorylation of GIT1 tyrosine 321 is required for association with FAK at focal adhesions and for PDGF-activated migration of osteoblasts. Mol Cell Biochem 365: 109-118, 2012

11. Ren K,Liu F, Huang Y, et al: Periodic mechanical stress activates integrin $\beta 1$-dependent Src-dependent PLC $\gamma 1$-independent Rac1 mitogenic signal in rat chondrocytes through ERK1/2. Cell Physiol Biochem 30: 827-842, 2012.

12. Crooke CE, Pozzi A and Carpenter GF: PLC-gamma1 regulates fibronectin assembly and cell aggregation. Exp Cell Res 315: 2207-2214, 2009.

13. Husain D, Meyer RD, Mehta M, et al: Role of c-Cbl-dependent regulation of phospholipase Cgamma1 activation in experimental choroidal neovascularization. Invest Ophthalmol Vis Sci 51: 6803-6809, 2010.

14. Hunter I, Mascall KS, Ramos JW and Nixon GF: A phospholipase $\mathrm{C} \gamma 1$-activated pathway regulates transcription in human vascular smooth muscle cells. Cardiovasc Res 90: 557-564, 2011.

15. Kayal RA, Siqueira M, Alblowi J, McLean J, Krothapalli N, Faibish D, Einhorn TA, Gerstenfeld LC and Graves DT: TNF-alpha mediates diabetes-enhanced chondrocyte apoptosis during fracture healing and stimulates chondrocyte apoptosis through FOXO1. J Bone Miner Res 25: 1604-1615, 2010.

16. Oreffo RO: Growth factors for skeletal reconstruction and fracture repair. Curr Opin Investig Drugs 5: 419-423, 2004.

17. Stolker JM, Spertus JA, McGuire DK, et al: Relationship between glycosylated hemoglobin assessment and glucose therapy intensification in patients with diabetes hospitalized for acute myocardial infarction. Diabetes Care 35: 991-993, 2012.

18. Coutts RD, Sah RL and Amiel D: Effects of growth factors on cartilage repair. Instr Course Lect 46: 487-494, 1997.

19. Verschure PJ, Joosten LA, van der Kraan PM and Van den Berg WB: Responsiveness of articular cartilage from normal and inflamed mouse knee joints to various growth factors. Ann Rheum Dis 53: 455-460, 1994.

20. Kumei Y, Akiyama H, Hirano M, et al: Space flight modulates signal transduction pathway of growth factor receptors in rat osteoblasts. Biol Sci Space 13: 142-143, 1999.

21. Pang J, Xu X, Getman MR, et al: $\mathrm{G}$ protein coupled receptor kinase 2 interacting protein 1 (GIT1) is a novel regulator of mitochondrial biogenesis in heart. J Mol Cell Cardiol 51: 769-776, 2011

22. Zhang S, Hisatsune C, Matsu-Ura $\mathrm{T}$ and Mikoshiba $\mathrm{K}$ : G-protein-coupled receptor kinase-interacting proteins inhibit apoptosis by inositol 1,4,5-triphosphate receptor-mediated $\mathrm{Ca}^{2+}$ signal regulation. J Biol Chem 284: 29158-29169, 2009. 